\title{
Characterization of Loaded Pre-stressed Piezoelectric Actuators
}

\author{
Karla Mossi ${ }^{1}$, Lisa Scott ${ }^{2}$, Shivan $\operatorname{Haran}^{3}$ \\ ${ }^{1}$ Virginia Commonwealth University, Richmond, VA 23284-3015 \\ ${ }^{2}$ Dominion Energy, Inc., Hampton, VA 23663-2206 \\ ${ }^{3}$ Arkansas State University, State University, AR 72467
}

\begin{abstract}
Over the past few years, actuators have been characterized and analyzed under several different boundary conditions. One of these conditions is loading, which plays a critical part in any application where the actuator is used in a structure. This study concentrated on comparing the displacement under load for different types of actuators of the same length and width, but having different layer compositions. The same load and excitation frequency was used at different voltages. Eleven different groups of devices were tested with metal thickness ratios (MTR - ratio of metal thickness to the total thickness) varying between 0.3 and 0.44 . The metals used were stainless steel and brass. The results indicated that the devices with an MTR between 0.31 and 0.37 were able to lift a load of 76 grams. All the devices with this MTR had a ceramic thickness of $0.38 \mathrm{~mm}$ and stainless steel or brass backing. It was also observed that the positive and negative peak displacements were equal for most devices except for the devices with a metal to PZT thickness ratio of 1 showing a negative peak displacement more than $65 \%$ higher than positive peak displacement. This may be due to differences in location of the neutral axis, distribution of pre-stresses, and deformations of the devices under load. It was found that the maximum displacement per applied voltage per $\mathrm{mm}$ of ceramic thickness was produced by the devices, which had an MTR of 0.33 to 0.37 , with no top, and with a ceramic thickness of $0.38 \mathrm{~mm}$.
\end{abstract}

\section{Introduction}

Pre-stressed piezoelectric laminate devices have demonstrated enhanced technological performance as actuators over the past several years $[1,2]$. These devices are relatively new and have been gaining wide spread interest for a broad range of applications $[3,4]$. There are several parameters that are required to determine the feasibility of incorporating a pre-stressed piezoelectric laminate device into a given application; the type and geometry of the materials selected, mounting and loading conditions, and environmental effects [5]. One of the theories developed, based on geometry, shows that the behavior of a pre-stressed piezoelectric device under prescribed conditions can be predicted by the use of a simple parameter [6]. This parameter, metal-to-thickness ratio (MTR), relates the metal thickness of the reinforcing material to the total thickness of the piezoelectric laminate device. MTR is a quick method that can readily be applied to predict the performance of unloaded devices made with stainless steel that are subjected to pin-free boundary conditions and with a beam like geometry. Loading conditions, however, are a critical parameter for any application of interest. Many of the initial studies on these devices focused on their performance having varying geometries and mounting conditions but few have factored in their performance when dynamically loaded [7-10]. Consequently, there is a significant amount of research ongoing to more accurately quantify their behavior under varying loading conditions [11].

This particular study focused on determining the displacement of the pre-stressed piezoelectric laminate under specific loading conditions. The devices were clamped at one end, and free at the other end, to simulate a simply supported system under a load of 76 grams applied to the center; measurements were taken over a wide voltage range at $5 \mathrm{~Hz}$. The reinforcing materials were changed resulting in a variance in the metal-to-thickness ratio (MTR). Devices having a MTR of 0.33 to 0.4 were selected since this was the range that generated the greatest displacement based on results from Ounaies et al [6]. Aluminum, brass and stainless steel metals were selected since they are materials commonly used in the fabrication of these devices. This paper highlights the results from this study and attempts to explain the behavior observed for the various MTR's employed. 


\section{Experimental Setup}

The pre-stressed piezoelectric devices were manufactured by Dominion Energy using PZT 3195HD type piezoelectric ceramic (CTS Wireless). The metal reinforcing materials were T6-Aluminum, 304 Stainless Steel and Yellow Brass (Small Parts). SI ${ }^{\mathrm{TM}}$ polyimide (Dominion Energy) was used to laminate the metal(s) to the PZT. The overall width and length of the devices was $7.62 \mathrm{~cm} \times 1.25 \mathrm{~cm}$. The device layer description, including metal and thickness, is outlined in Table 1.

Table 1. Devices Layer Composition and Thickness

\begin{tabular}{|c|c|c|c|c|c|c|c|c|}
\hline \multirow{2}{*}{ ID } & Material & \multicolumn{2}{|c|}{ TOP } & \multicolumn{2}{c|}{ PZT } & \multicolumn{2}{c|}{ BOTTOM } & Material \\
\cline { 2 - 9 } & Type & mils & $\mu \mathrm{m}$ & mils & $\mu \mathrm{m}$ & mils & $\mu \mathrm{m}$ & Type \\
\hline 1 & $\mathrm{Al}^{1}$ & 1 & 25.4 & 15 & 381.0 & 10 & 254 & $\mathrm{SS}^{2}$ \\
\hline 2 & None & 0 & 0.0 & 15 & 381.0 & 10 & 254 & $\mathrm{SS}$ \\
\hline 3 & None & 0 & 0.0 & 7 & 177.8 & 5 & 127 & $\mathrm{SS}$ \\
\hline 4 & None & 0 & 0.0 & 10 & 254.0 & 10 & 254 & $\mathrm{SS}$ \\
\hline 5 & None & 0 & 0.0 & 15 & 381.0 & 10 & 254 & $\mathrm{BR}^{3}$ \\
\hline 6 & None & 0 & 0.0 & 7 & 177.8 & 6 & 152 & $\mathrm{BR}$ \\
\hline 7 & None & 0 & 0.0 & 10 & 254.0 & 10 & 254 & $\mathrm{BR}$ \\
\hline
\end{tabular}

${ }^{1}$ Aluminum Type T6; ${ }^{2}$ Stainless Steel $304 ;{ }^{5}$ Yellow Brass

Quality assurance was performed using a Philips MGC3 x-ray machine to ensure there were no cracks in the devices prior to testing. The thickness measurements were taken as an average of three points along the length of the device using a Multitoyo caliper. A NAIS LM10 non-contact laser was used to measure the dome height of each device. Dome height is defined as the maximum point in the curvature of the device. It is believed to be a result of the amount of pre-stress imparted due to the manufacturing process. Capacitance was measured at a frequency of $60 \mathrm{~Hz}$ using a Fluke 189 multimeter.

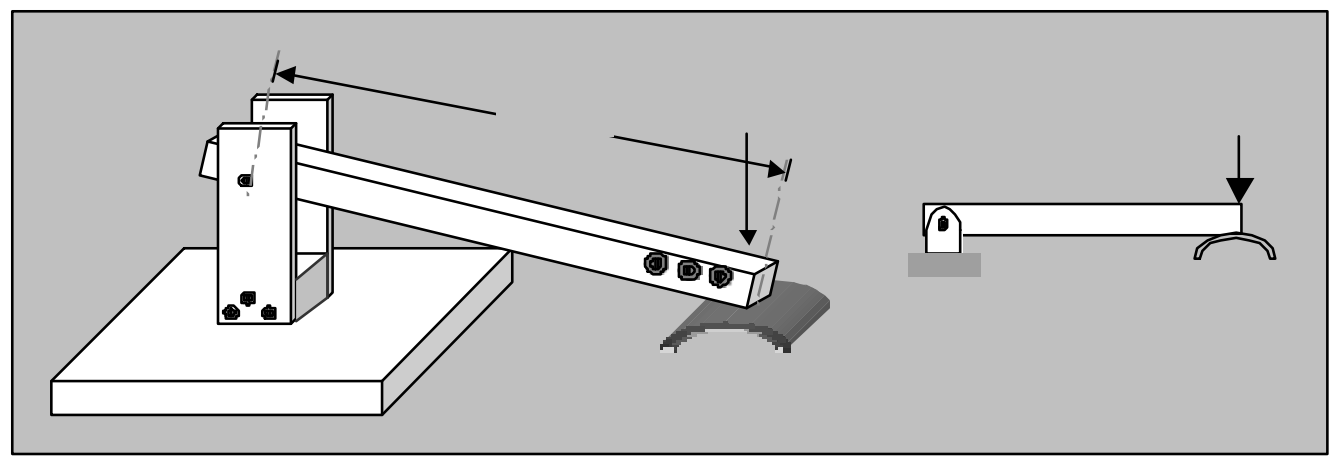

Figure 1. Test Set-up

The test apparatus was set up in the configuration illustrated in Figure 1. Displacement was measured under voltages ranging from 200Vp -p to 900Vp-p at $5 \mathrm{~Hz}$ using Trek PZD200 and Tektronics PS280 power supplies in conjunction with a HP 3320 signal generator, LeCroy 9350L oscilloscope, and NAIS LM10 laser. All instrumentation was controlled by a PC through routines written in LabView. 


\section{Results and Discussion}

Total thickness, dome height, and capacitance were measured for all the devices prior to the displacement performance tests. These measurements are used for reference and at the same time to provide some quality control of the devices. Also, material thickness ratio was calculated for each device composition. For this particular study, the MTR index was found to be in the range of 0.3 to 0.4 , which by previous studies, as illustrated in Figure 2 , indicates these are the devices that should produce the most displacement. It is important to note that the previous study tested devices made with the same metal (stainless steel) and the devices were measured under free displacement, i.e. unloaded; the devices in this study were tested under a $76 \mathrm{~g}$ load along with different metals. According to the results shown, also in Figure 2, the highest dome height should produce the most displacement, which ranged between 0.305 and $0.356 \mathrm{~mm}(120-140$ mils). The highest dome-height was measured for group numbers 3 and 6, followed by 5 and 7; Groups 3 and 6 had the thinnest construction. Dome heights for groups 3, 5, 6 and 7 were approximately $30 \%$ higher than the rest of the groups and are twice the predicted values in figure $1(>0.6 \mathrm{~mm})$. A summary of these results is shown in Table 2 .

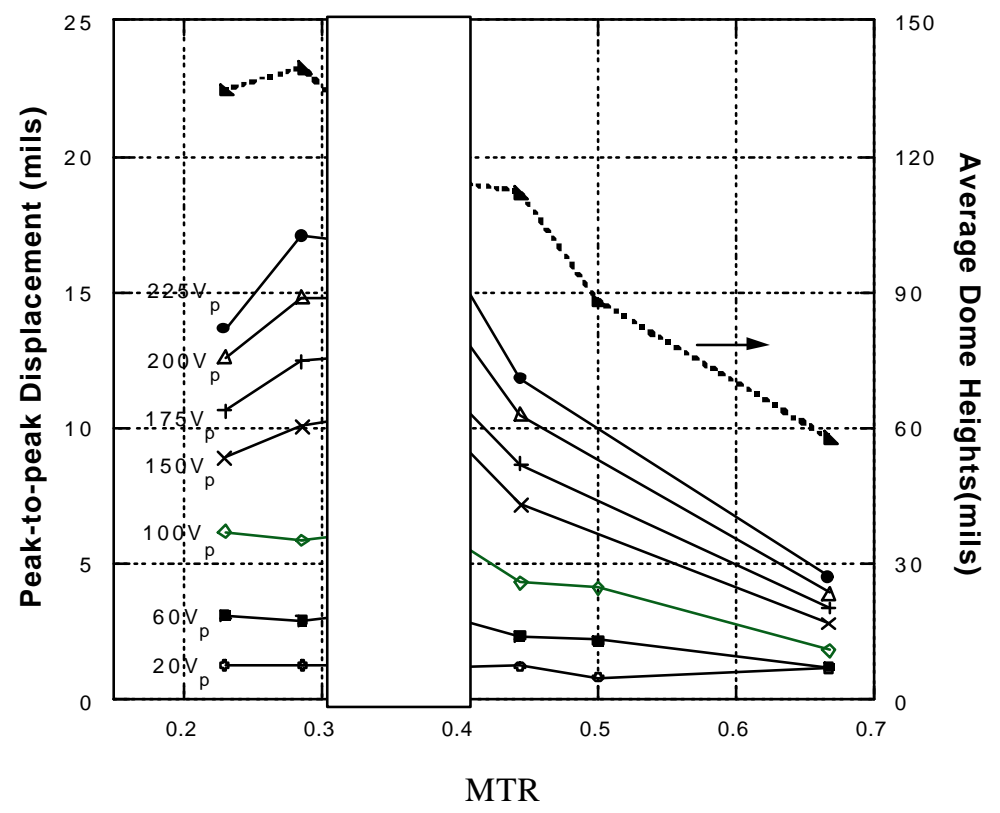

Figure 2. Metal-to-Thickness Ratio

Another parameter measured was capacitance at $60 \mathrm{~Hz}$. This parameter is used to calculate the dielectric constant of the devices to ensure conductivity throughout all the layers. These results are also shown in Table 2.

The devices were then tested for loaded displacement at 5 Hzin a simply supported manner at voltages ranging from $200 \mathrm{Vp}$ - $\mathrm{p}$ to $900 \mathrm{Vp}$ - $\mathrm{p}$. The displacement was monitored and recorded in a voltage displacement loop, see Figure 3 for a typical loop at $5 \mathrm{~Hz}$ for two different groups. Some of the devices belonging to groups 3 and 6 could not be measured since the load applied produced deformed loops. Furthermore, the samples belonging to these groups failed under the 76-gram load. This was most likely due to the thinner construction of the devices.

Note that the loops show peak displacement at voltages lower than the maximum/minimum voltages applied. That is, the highest voltage is not the only place that produces the most displacement. Hence, smaller voltages can be applied (DC) to obtain the same amount of displacement at low frequencies with a load of 76 grams. 
Table 2. Physical Parameters and Material Thickness Ratio

\begin{tabular}{|c|r|c|c|c|c|c|}
\hline \multirow{2}{*}{ ID } & \multicolumn{2}{|c|}{ Total Thickness } & \multicolumn{2}{c|}{ Dome Height } & Capacitance & \multirow{2}{*}{ MTR } \\
\cline { 2 - 6 } & $\mathbf{( m i l s )}$ & $(\mathbf{m m})$ & $\mathbf{m i l s})$ & $(\mathbf{m m})$ & $(\mathbf{n F})$ & \\
\hline 1 & 31.99 & 0.813 & 172.4 & 0.438 & 25.9 & 0.313 \\
\hline 2 & 27.92 & 0.709 & 193.3 & 0.491 & 34.9 & 0.358 \\
\hline 3 & 16.89 & 0.429 & 303.9 & 0.772 & 41.0 & 0.296 \\
\hline 4 & 22.61 & 0.574 & 192.1 & 0.488 & 41.2 & 0.442 \\
\hline 5 & 30.62 & 0.778 & 247.6 & 0.629 & 32.5 & 0.327 \\
\hline 6 & 18.68 & 0.474 & 264.2 & 0.671 & 62.1 & 0.321 \\
\hline 7 & 25.83 & 0.656 & 247.6 & 0.629 & 45.3 & 0.387 \\
\hline
\end{tabular}

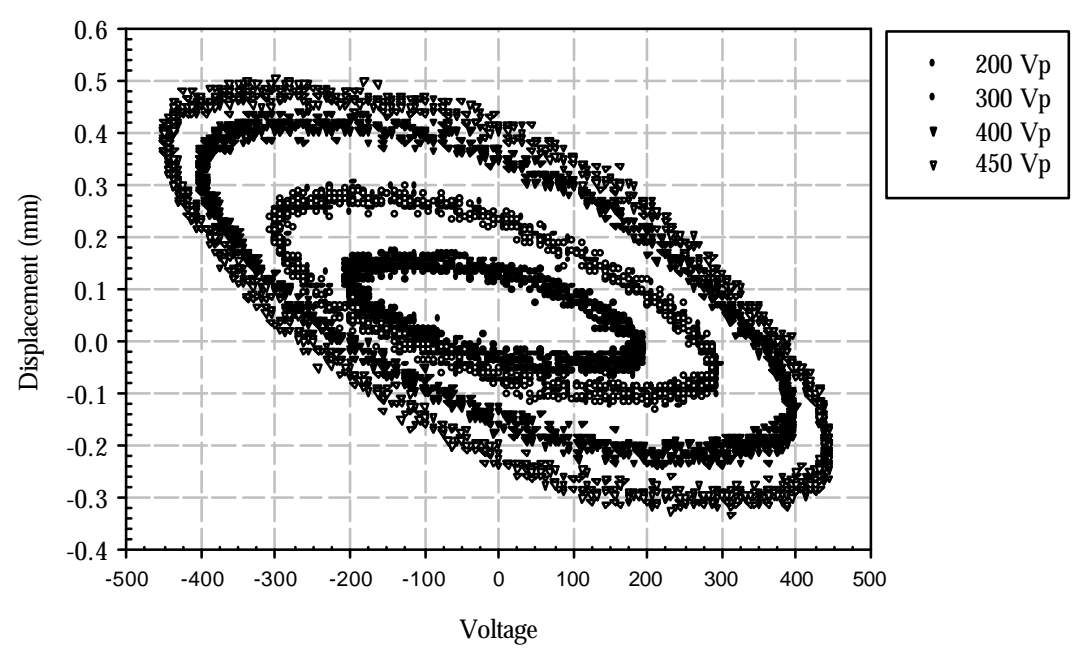

(a) Group 1

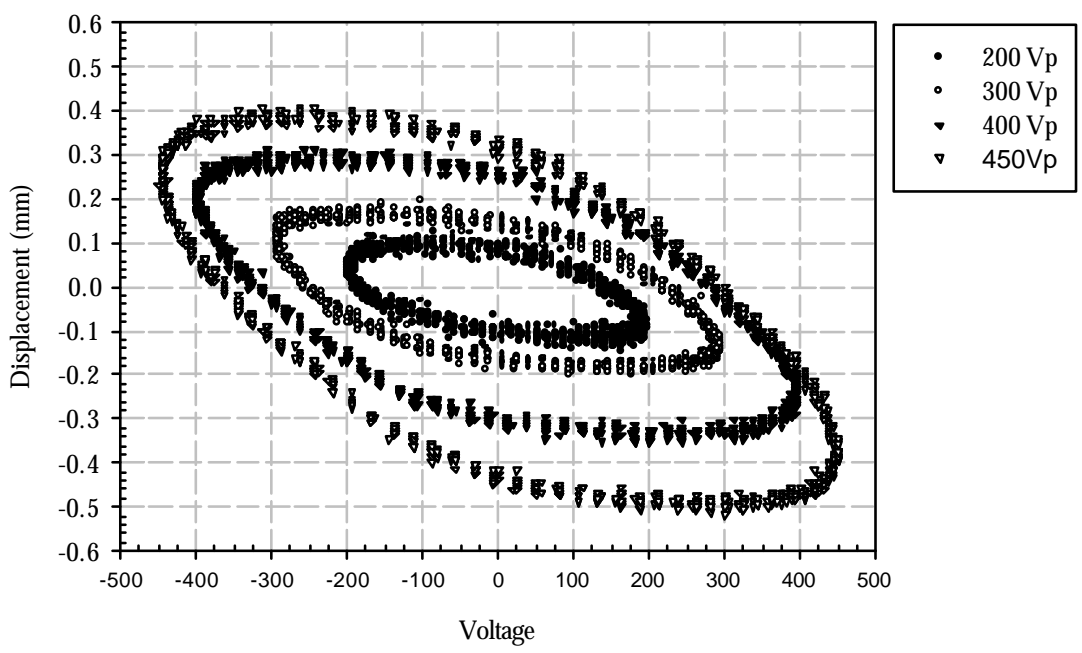

(b) Group 5

Figure 3. Voltage Displacement loop for a typical sample 
In order to compare all of the pieces, maximum and minimum peak displacements were taken from all the loops measured at $5 \mathrm{~Hz}$. The results were averaged for the groups that could be measured and are plotted in Figure 4 . It is interesting to note that the performance trend for group 1, the pieces with an aluminum top layer, was similar to the best performers of the groups and exhibited almost as much displacement as the pieces that had no top layer. See Figure 5 below for an enhanced view of these results. This indicates the aluminum is not restricting the motion of the device and adversely affecting the performance. Being able to construct devices using the aluminum as a top layer has an advantage in that it makes it easier to attach leads to the top of the device. Groups 2 and 5 were the best performers of all the groups producing maximum and minimum displacements that were very similar. These two groups had the same layer configurations, but different backing materials, stainless steel and brass of the same thickness; however, their respective dome heights in this case were different by more than $20 \%$, making dome-height alone a parameter that cannot be used to determine performance. However, the parameter, MTR, did accurately predict the performance of the devices under loaded conditions. Other important factors to consider is the asymmetry of the displacement produced; Groups 1,2, and 5 are fairly symmetric - that is positive and negative displacement; Groups 4 and 7 move more than $65 \%$ of the total displacement in the negative direction. This indicates the pieces are weaker and cannot lift the 76 grams load.

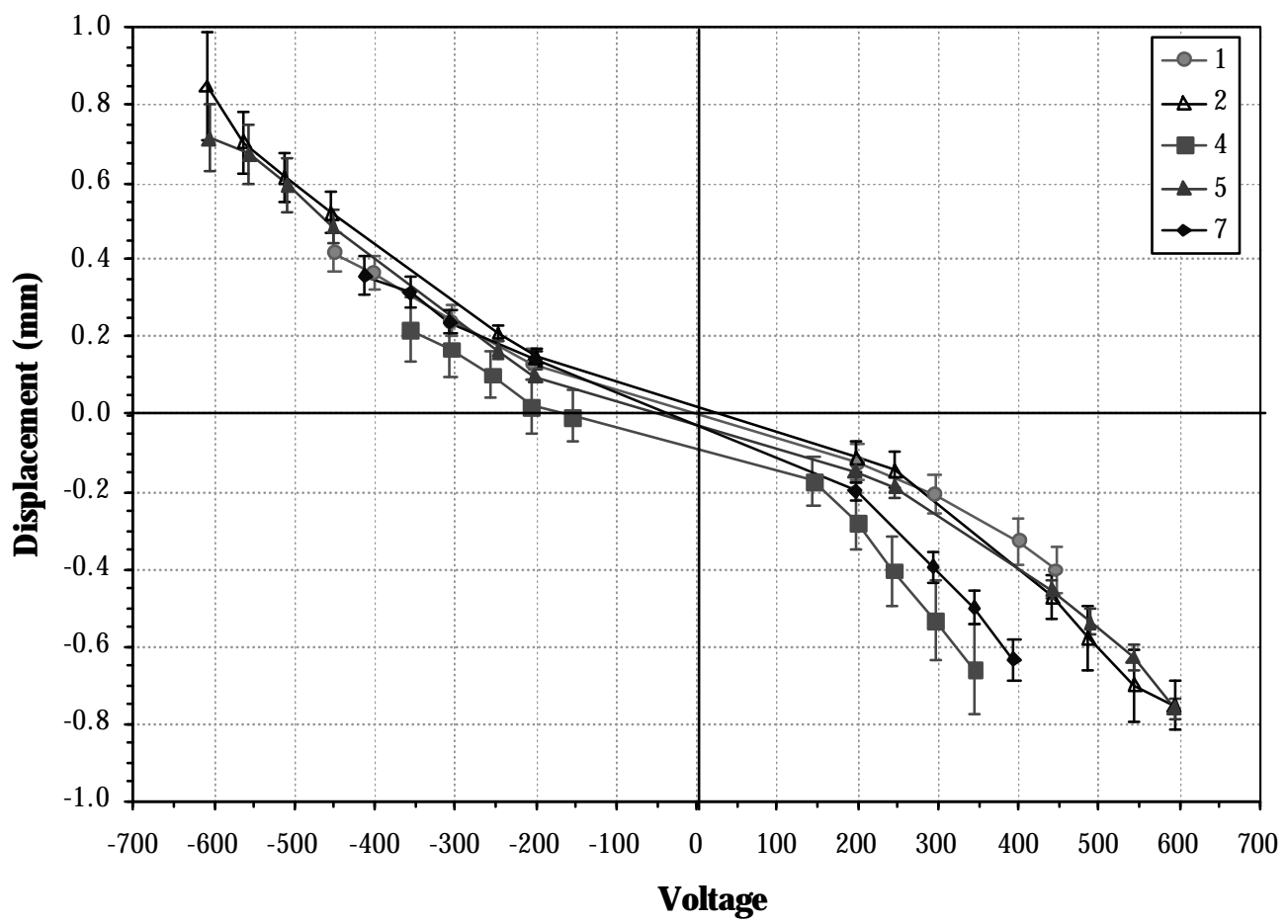

Figure 4. Average Maximum and Minimum Peak Displacement for All Groups at $\mathbf{5 H z}$

The peak to peak displacement for each of the groups was plotted as a function of the voltage per millimeter of PZT and adhesive line, that is total displacement per field applied, see Figure 6. The group that performed the best was group number 5 , which is a device made of brass. This group is closely followed by 2 which has identical layer setup but fabricated with stainless steel. The maximum overall displacement was in the range of 1.3 to $1.6 \mathrm{~mm}$ produced with fields higher than $2000 \mathrm{~V} / \mathrm{mm}$, MTR of 0.358 and 0.327 , however, the dome heights varied from 0.491 and 0.629 with the devices of group 5 having the greatest dome height. 


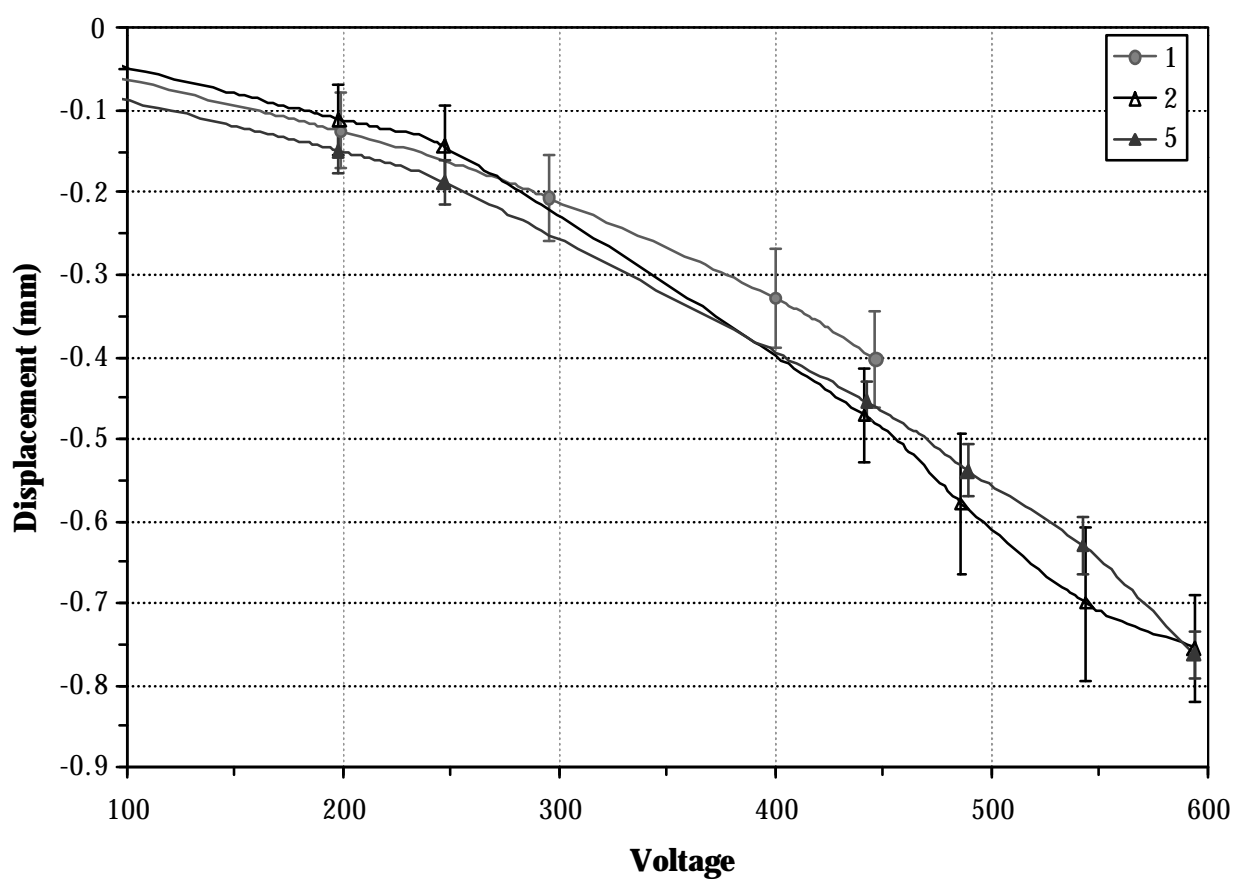

Figure 5. Average Maximum and Minimum Peak Displacement for Selected Groups at 5Hz

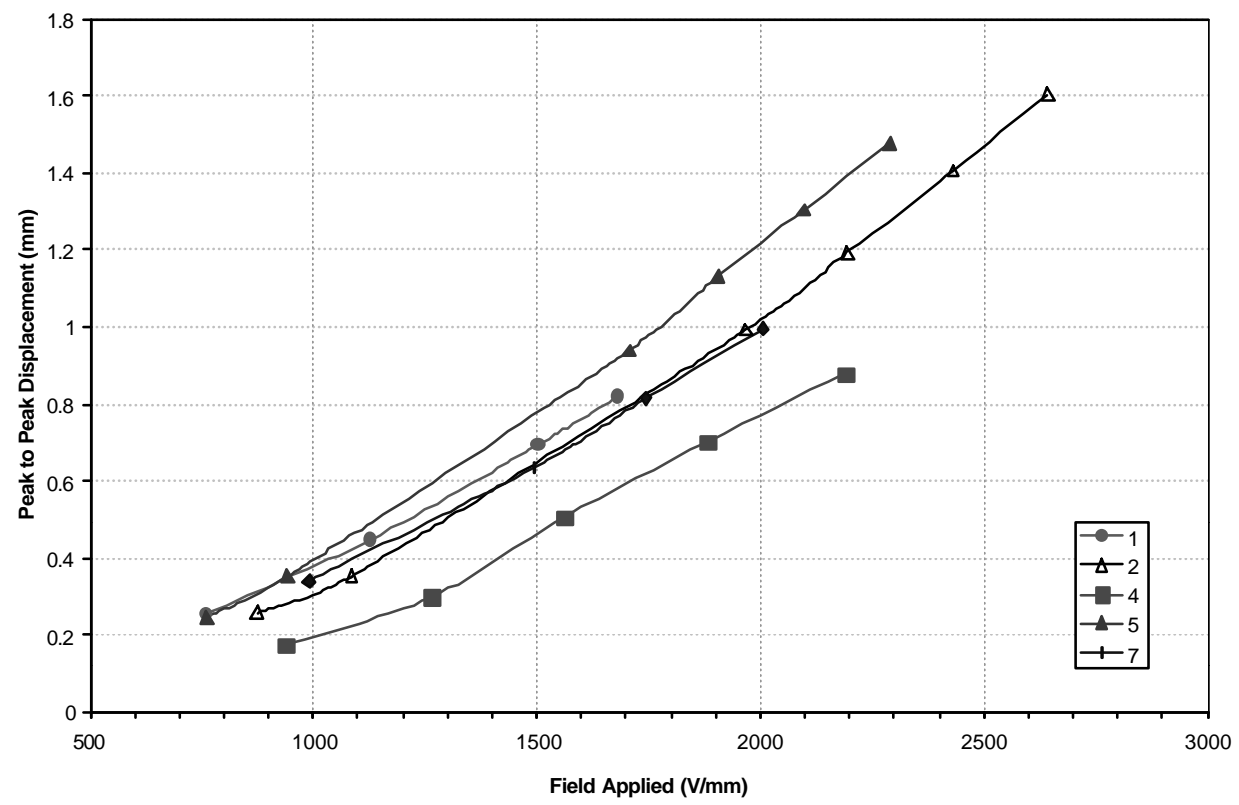

Figure 6. Total Displacement as a Function of Applied Field 


\section{Conclusions}

Seven groups of pre-stressed piezoelectric laminate devices, having different layers thickness and materials, were tested to determine their performance under a $76 \mathrm{gram}$ load over a wide range of voltages. Devices having $0.381 \mathrm{~mm}$ PZT and $0.254 \mathrm{~mm}$ brass backing material produced the greatest displacement under load at $5 \mathrm{~Hz}$. This group was closely followed by the same layer configuration with stainless steel backing. It was found for all of the groups that devices with dome heights $20-30 \%$ greater did not necessarily produce the maximum displacement. It was also found that applying higher AC voltages when trying to lift a load not necessarily produce more displacement. The same amount of displacement can be produced with a lower DC voltage. It was also observed that the positive and negative peak displacements were equal for most devices except for the devices with a metal to PZT thickness ratio of 1 showing a negative peak displacement more than $65 \%$ higher than positive peak displacement. This may be due to differences in location of the neutral axis, distribution of pre-stresses, and deformations of the devices under load - inability to support the 76 grams load.

The MTR theory and dome height measurements were applied to determine if it would be applicable in predicting the performance of devices having different metal backings under load. Results show that dome height alone cannot be used to predict the performance of devices under loaded conditions. It was found that the devices having a MTR between 0.31 and 0.37 were able to lift the 76 grams load and also produced the most displacement. These results were found to correspond well with the MTR theory.

\section{Acknowledgements}

This project was supported by Dominion Resources Inc. under VCU contract number 02-0051-00.

\section{References}

1. Li. G., Furman, G., and Haertling, G. "Stress-enhanced displacement in PLZT Rainbow actuators," Journal of the American Ceramic Society, Vol. 80, 1997.

2. Mossi, K., Selby, G., and Bryant, R. “"Thin-layer composite unimorph ferroelectric driver and sensor properties," Materials Letters, Vol. 35, 1998.

3. Munday, D., and Jacob, J. "Active Control of Separation on a Wing with Conformal Chamber," $39^{\text {th }}$ AIAA Aerospace Sciences Meeting and Exhibit, January 8-11, 2001/ Reno, NV.

4. Lobontiu, N., Goldfarb, M., Garcia, E. "A piezoelectric-driven inchworm locomotion device," Mechanism and Machine Theory 36 (2001) 425-443

5. Schwartz, R., Narayanan, M. "Development of high performance stress-biased actuators through the incorporation of mechanical pre-loads," Sensors and Actuators A 101 (2002) 322-331.

6. Ounaies, Z., Mossi, K., Smith, R., Berndt, J. " Low-Field and High Field Characterization of Thunder Actuators," SPIE 2001 Conference, 4333-66, March 2001.

7. Shakeri, C., Bordonaro, C., Noori, M., and Champagne, R. "Experimental Study of THUNDER: A New Generation of Piezoelectric Actuators," Proc. of SPIE Conference, Newport Beach, CA, 1-5 March, vol. 3675-07.

8. Hyer, M.W., and Jilani A. " Predicting the Deformation Characteristics of Rectangular Unsymmetrically Laminated Piezoelectric Materials," Smart Materials and Structures, 7: p.784-791.

9. Wise, S.A. " Displacement properties of RAINBOW and THUNDER piezoelectric actuators," Sensors and Actuators A, Vol. 69, 1998.

10. Wieman, R., Smith, R., Ounaies, Z., and Bernd, J. "A displacement model for thunder actuators having general loads and boundary conditions", SPIE Proceedings: Modeling, Signal Processing and Control in Smart Structures, Vol. 4326, 2001.

11. Mulling, J., Usher, T., Dessent, B., Palmer, J., Franzon, P., Grant, E., and Kingon, A. "Load characterization of high displacement piezoelectric actuators with various end conditions," Sensors and Actuators A: Physical, v.94: pp 19-24. 